\title{
ARE SUPERSOFT X-RAY SOURCES CONSISTENT WITH WHITE DWARFS?
}

\author{
A. VAN TEESELING \\ Universitäts-Sternwarte Göttingen \\ Geismarlandstr. 11, D-37083 Göttingen \\ J. HEISE \\ SRON Laboratory for Space Research \\ Sorbonnelaan 2, NL-3584 CA Utrecht \\ AND \\ P. KAHABKA \\ Astronomical Institute "Anton Pannekoek" \\ Kruislaan 403, NL-1098 SJ Amsterdam
}

\section{Introduction}

Van den Heuvel et al. (1992) argued that the emission from supersoft X-ray sources can be explained by steady nuclear burning of hydrogen accreted onto massive white dwarfs. An argument that has been used against this model is that the bolometric luminosities inferred from blackbody fits are sometimes above the Eddington luminosity of a $\sim 1 \mathrm{M}_{\odot}$ star. However, hot white dwarfs don't radiate blackbody spectra (Heise et al. 1994). Therefore, we have calculated model spectra for hot (accreting) white dwarfs, and investigated whether the emission from supersoft $\mathrm{X}$-ray sources is consistent with an origin from white dwarfs. Here we summarize some preliminary results. For a review of supersoft $\mathrm{X}$-ray sources we refer to the contribution of Kahabka \& Trümper to these proceedings.

\section{LTE White-Dwarf Model Atmospheres}

Fig. 1 shows a selection of the calculated spectra for LMC abundances. The spectra are almost flat up to a strong absorption edge of hydrogen-like or helium-like $\mathrm{C}, \mathrm{N}$, or $\mathrm{O}$. Our model atmospheres emit relatively more 


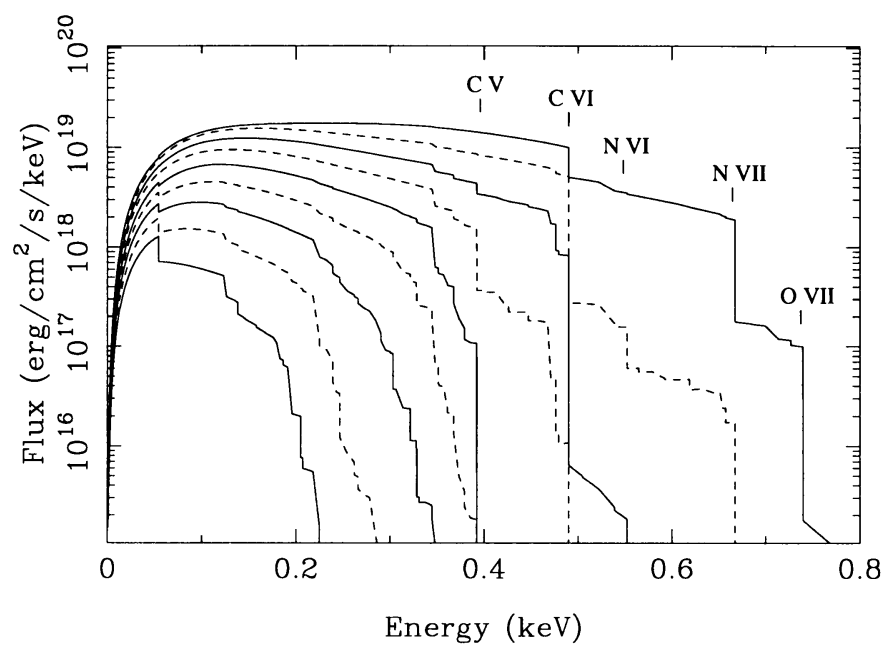

Figure 1. Emergent fluxes of LTE white-dwarf model atmospheres for $0.25 \times$ cosmic abundances, $\log g=8$, and $T_{\text {eff }}=210^{5} \mathrm{~K}$ to $610^{5} \mathrm{~K}$ in steps of $510^{4} \mathrm{~K}$ from bottom to top.

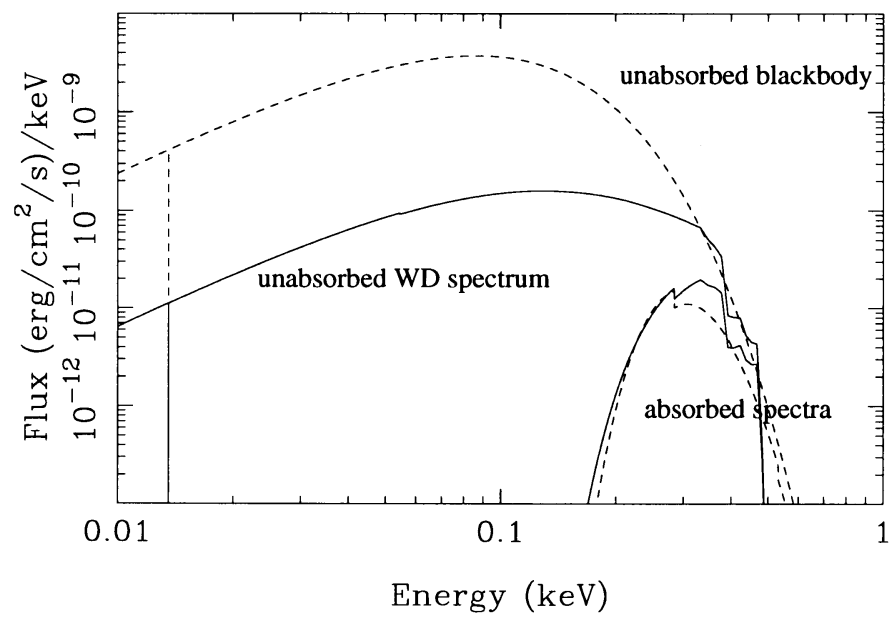

Figure 2. The best-fit blackbody spectrum and the best-fit $\log g=8$ white-dwarf model atmosphere spectrum of the supersoft X-ray source $1 \mathrm{E} 0056.8-7154$.

soft $\mathrm{X}$-rays than blackbodies. This shows that very hot white-dwarfs are predominantly soft $\mathrm{X}$-ray sources.

To illustrate the effect of fitting white-dwarf model atmosphere spectra instead of blackbody spectra to the observed spectra of supersoft X-ray sources, we first consider $1 \mathrm{E} 0056.8-7154$. This source has been identified with the planetary nebula N67 in the SMC (Wang 1991). Fig. 2 shows the 

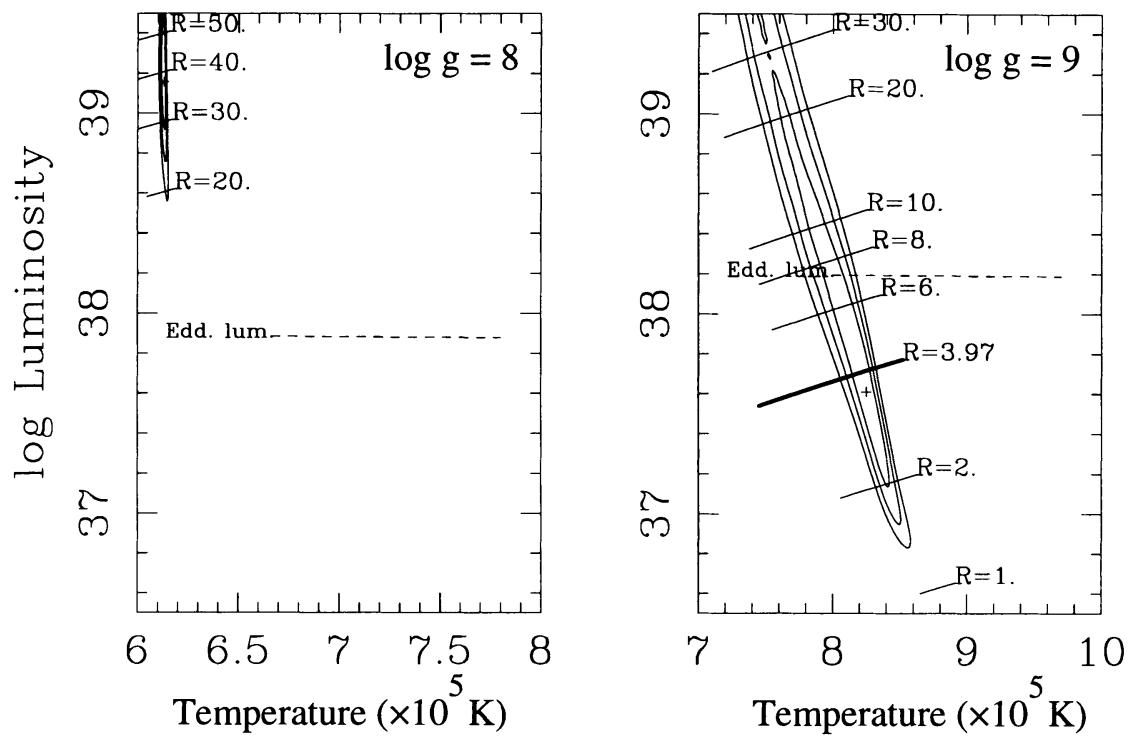

Figure 3. $\chi^{2}$ contours at 1,2 and 3- $\sigma$ levels for fits of model atmospheres to the spectrum of CAL 87. The bolometric luminosity is given in $\operatorname{erg~s}^{-1}$. Contours of equal normalization constant are labeled with the radius $\left(\times 10^{8} \mathrm{~cm}\right)$ of the source at $52 \mathrm{kpc}$. The thick line is for the normalization constant corresponding to the radius of a non-expanded white dwarf with the $\log g$ of the models.

best-fit blackbody spectrum and the best-fit $\log g=8$ model atmosphere spectrum for the ROSAT spectrum of 1E 0056.8-7154. The unabsorbed bolometric luminosity of the model atmosphere $\left(210^{37} \mathrm{erg} \mathrm{s}^{-1}\right)$ is well below the Eddington luminosity of $a \sim 1 \mathrm{M}_{\odot}$ star and more than a factor of ten lower than the luminosity of the blackbody.

\section{Inferred Luminosities and Radii}

The spectral resolution of the ROSAT PSPC is not sufficient to discriminate between blackbody spectra and model atmosphere spectra. Every spectrum that has a steep cut off at $\sim 0.5 \mathrm{keV}$ will fit the observed ROSAT spectra of supersoft X-ray sources. However, for a particular type of star (e.g., a white dwarf) the inferred radius has to be consistent with the gravity used to calculate the model atmospheres. For $1 \mathrm{E} 0056.8-7154$ the inferred radius is indeed consistent with a massive $\log g=8$ white dwarf.

To investigate whether other supersoft $\mathrm{X}$-ray sources, for which it is not certain that they contain a white dwarf, are also consistent with white dwarfs, we analysed ROSAT PSPC observations of seven other supersoft $\mathrm{X}$-ray sources in the Magellanic Clouds. 

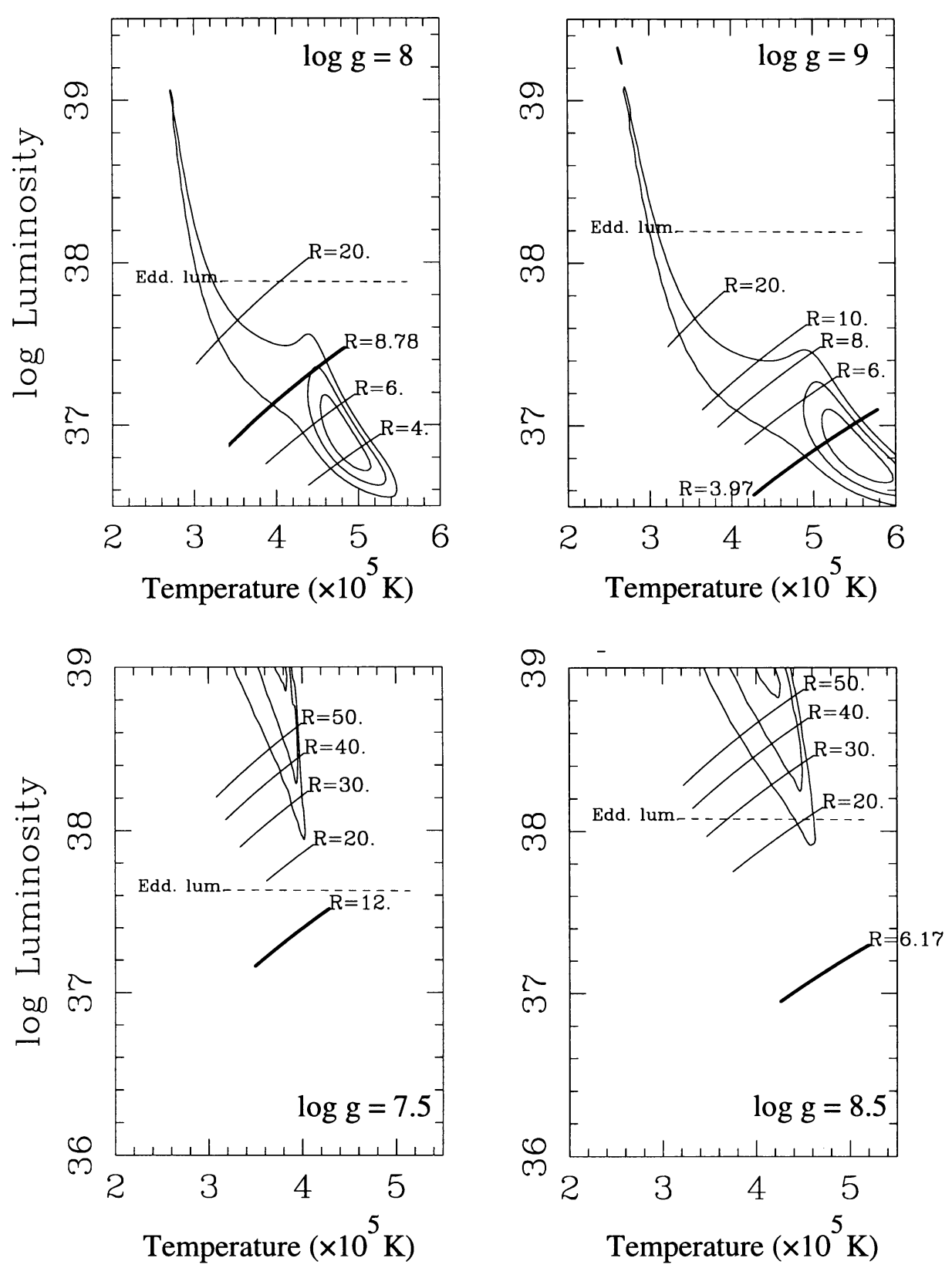

Figure 4. 1, 2, and 3- $\sigma$ contours for 1E 0035.4-7230 (top panels), and RX J0048.4-7332 (bottom panels). The bolometric luminosities are given in $\mathrm{erg} \mathrm{s}^{-1}$. Lines of equal inferred radii are given in units of $10^{8} \mathrm{~cm}$. The Eddington luminosities and the thick lines correspond to the radii of non-expanded white dwarfs with the appropriate $\log g$. 
Fig. 3 shows the confidence levels for CAL 87. It is not possible to get a consistent fit with $\log g=8$, because the inferred radii are too large for a white dwarf. However, CAL 87 has a harder spectrum than most other supersoft X-ray sources, indicating a high effective temperature. This is possible with larger gravities. Indeed, the spectrum and count rate of CAL 87 are consistent with a $\log g=8.5$ and $\log g=9$ white dwarf.

Fig. 4 shows the $\chi^{2}$ contours for $1 \mathrm{E} 0035.4-7230$ in the SMC. The observations are consistent with both $\log g=8$ and $\log g=9$ LTE atmospheres. For seven of the analysed sources we find radii which are consistent with white dwarfs. The only exception is RX J0048.4-7332 in the SMC. For all gravities the inferred radii are significantly larger than the radii of nonexpanded white dwarfs (Fig. 4). RX J0048.4-7332 has been identified with the symbiotic system SMC3 (Kahabka et al. 1994), which probably had a significant increase in luminosity in 1981 (Vogel \& Morgan 1994).

\section{Steady Nuclear Burning of Accreted Hydrogen}

In Fig. 5 we have plotted for the five analysed LMC sources the 1- $\sigma$ confidence contours. The observed spectra are consistent with LTE white-dwarf spectra within these contours. Also, within these contours the inferred radii are consistent with a not significantly expanded white dwarf. The elongated shape of the contours is due to the fact that a spectrum for higher gravity and temperature is similar to a spectrum for lower gravity and lower temperature. CAL 87 is consistent with a high-mass white dwarf with a high effective temperature. RX J0439.8-6809 is consistent with an intermediatemass white dwarf with a lower effective temperature.

\section{Conclusions}

LTE model atmosphere spectra may give bolometric luminosities of a factor of ten lower than blackbody spectra. For seven of the eight analysed supersoft X-ray sources the inferred best-fit luminosities are below the Eddington luminosity of a solar mass star. The derived radii of these sources are consistent with white dwarfs, and the bolometric luminosities and effective temperatures are consistent with white dwarfs with steady nuclear burning of accreted hydrogen. We did not prove that supersoft $\mathrm{X}$-ray sources are white dwarfs, but our analysis indicates that the X-ray observations of these sources can be explained with emission from hot white dwarfs. 


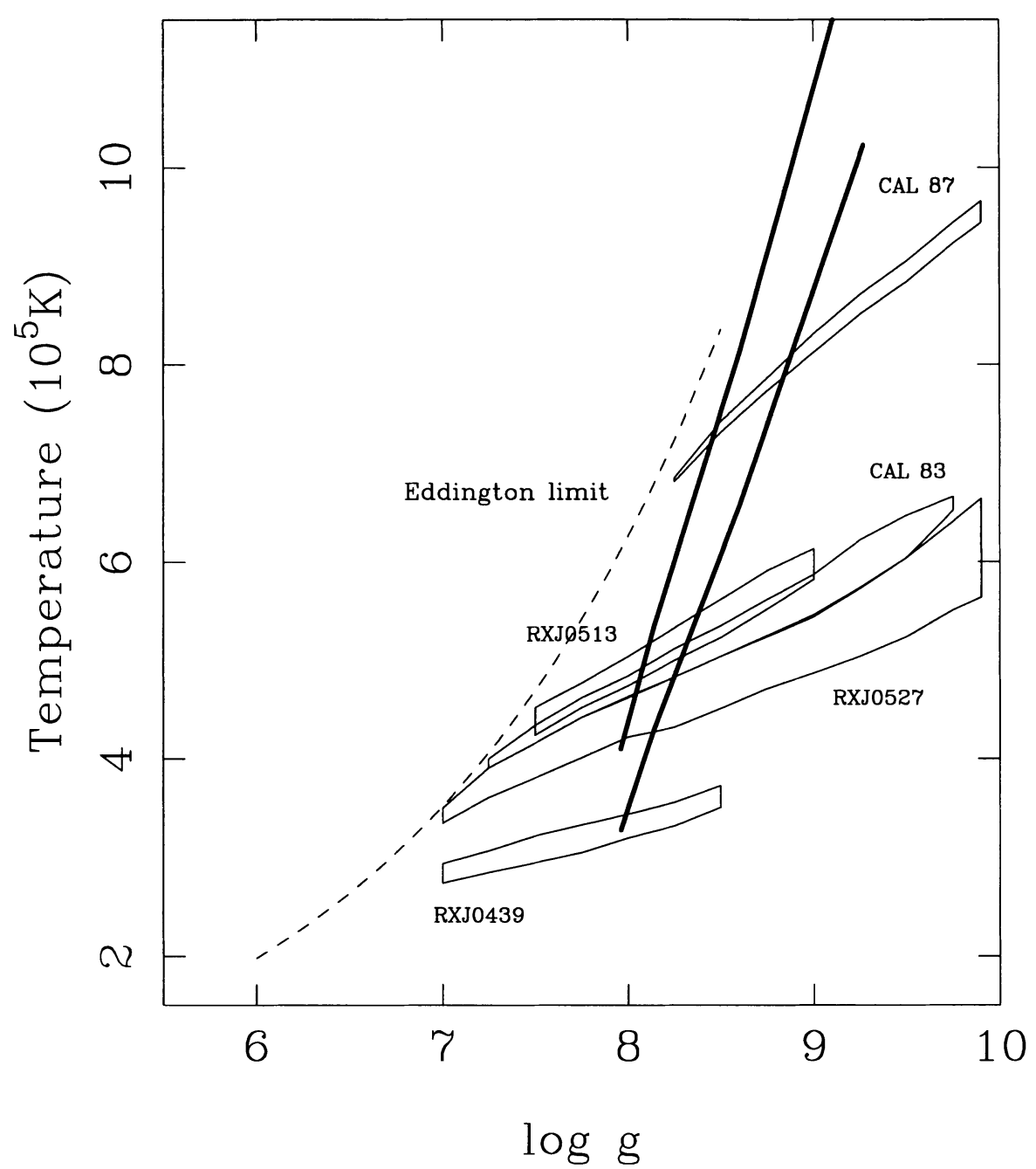

Figure 5. Results of fitting LTE model atmospheres to supersoft X-ray sources in the LMC. The 1- $\sigma$ range of effective temperatures and $\log g$ for which self-consistent model fits can be obtained is plotted. The solid thick lines bound the region where steady nuclear burning of hydrogen on non-expanded white dwarfs with masses larger than $0.6 \mathrm{M}_{\odot}$ is stable (Van den Heuvel et al. 1992).

\section{References}

Heise, J., Van Teeseling, A. \& Kahabka, P. 1994, A\&A 288, L45

Kahabka, P., Pietsch, W. \& Hasinger, G. 1994, A\&A 288, 538

Van den Heuvel, E.P.J. et al. 1992, A\&A 262, 97

Vogel, M. \& Morgan, D.H. 1994, A\&A 288, 842

Wang, Q. 1991, MNRAS 252, 47P 